\title{
Factors affecting time to resolution of suicidality for depressed patients receiving electroconvulsive therapy
}

\author{
Ching-Hua Lin, ${ }^{1,2 *}$ (1) Wei-Cheng Yang, ${ }^{\prime}$ and Jyh-I Wu ${ }^{3}$
}

\author{
${ }^{1}$ Kaohsiung Municipal Kai-Syuan Psychiatric Hospital, Kaohsiung, Taiwan \\ ${ }^{2}$ Department of Psychiatry, School of Medicine, College of Medicine, Kaohsiung Medical University, Kaohsiung, Taiwan \\ ${ }^{3}$ Department of Anesthesiology, Kaohsiung Municipal Min-Sheng Hospital, Kaohsiung, Taiwan
}

Received 7 March 2019; Accepted 7 March 2019; First published online 14 May 2019

Key words: major depressive disorder, electroconvulsive therapy, suicidality, past suicide attempt, suicide item.

To the editor:

The Diagnostic and Statistical Manual of Mental Disorders, Fourth Edition, Text Revision (DSM-IV-TR) or the Diagnostic and Statistical Manual of Mental Disorders, Fifth Edition (DSM-5) identifies suicidality, which encompasses suicidal thought, suicidal ideation, suicidal plan, or suicidal behavior, as one criterion of a major depressive disorder (MDD). Electroconvulsive therapy (ECT) rapidly resolves the suicidal symptoms in MDD patients. ${ }^{1}$ The determination of predictors of suicidal resolution can help clinicians target those that can be modified. We aimed to determine which baseline clinical variables affected the number of ECT treatments to suicidal resolution for MDD patients.

The study was the secondary analysis of an open-label trial conducted from January 2008 to October 2013 for depressed inpatients treated with ECT. ${ }^{2}$ The trial was approved by Kai-Syuan Psychiatric Hospital's institutional review board. Acutely ill MDD inpatients $(n=130)$ requiring ECT were enrolled if they were aged 18 years or more, satisfied DSM-IV-TR criteria for MDD, had a 17-item Hamilton Rating Scale for Depression (HAMD-17) $\geq 18$ at baseline, and had given written informed consent. Patients remained medication-free during the ECT course, with the exception of anxiolytic and sedative-hypnotic medications p.r.n. for insomnia or severe anxiety. Standard bilateral frontotemporal and modified ECT was performed. Seizure duration was at least $20 \mathrm{~s}$, as measured by electromyogram. The number of ECT treatments was determined by the treating psychiatrist, and based on the patient's response [i.e. whether remission (HAMD-17 $\leq 7$ ) was achieved], tolerance of side effects,

*Address correspondence to: Ching-Hua Lin, Department of Adult Psychiatry, Kaohsiung Municipal Kai-Syuan Psychiatric Hospital 130, Kai-Syuan $2^{\text {nd }}$ Rd., Ling-Ya District, Kaohsiung 802, Taiwan. (Email: chua.lin@msa.hinet.net) and decision to withdraw. ${ }^{3}$ The maximum number of ECT treatments was 12. Clinical characteristics including sex, past suicide attempt, treatment-resistant depression, substance abuse/dependence, psychotic features, ECT frequency (twice-weekly or thrice-weekly), age, duration of the current episode, and number of previous major depressive episodes were gathered at baseline (Supplementary material Table S1). Past suicide attempt was defined as a history of self-injurious act with an intent to die. Treatment-resistant depression was defined as a lack of response to two or more adequate trials of different classes of antidepressants. The level of hopelessness experienced during the past week was rated using the item 14 of the Zung Self-Rating Depression Scale. Pain over the past 1 month was measured by the Body Pain Index of SF-36. The Work and Social Adjustment Scale (WSAS) ${ }^{4}$ was used to measure the level of psychosocial functional impairment. The level of suicidality was rated using the HAMD-17 suicide item (Item 3) throughout the acute treatment. A score at least 1 was regarded as suicidality. Symptom severity and the level of suicidality were assessed using HAMD-17 by independent board-certified psychiatrists prior to initiating ECT treatment (baseline), after every third ECT treatment, and after the final ECT treatment. HAMD-17 was conducted 1-2 days after treatment. Analysis was done on a modified intent-to-treat basis. Subjects reporting at least one post-baseline assessment and baseline suicide item score $\geq 1$ (i.e. Item 3 score $\geq 1$ ) were included for the analysis. Statistical significance was set at $p<0.05$. Effect size was used to measure sensitivity to change at endpoint for the suicide item. The univariate Cox regression analysis was used to investigate statistically significant predictors affecting number of ECT treatments to suicidal resolution (i.e. from suicide item score $\geq 1$ to suicide item $=0$ ). If statistically significant predictors were obtained, they were entered into the forward multivariate 
Cox regression analysis to identify definite predictors adjusted for other covariates.

Among 116 patients receiving at least three ECT treatments, 111 patients with HAMD-17 item 3 score $\geq 1$ were included in the analysis (Supplementary material Figure S1). Supplementary material Table S1 shows the clinical variables at baseline. Ninety-two $(\mathbf{8 2 . 9 \% )}$ reached suicidal resolution after an acute treatment with ECT. The median time to suicidal resolution was 6.0 ECT treatments $(\mathrm{SE}=0.2)$. The analysis using univariate Cox regression analysis revealed that past suicide attempt, longer duration of the current episode, lower BPI scores, greater baseline WSAS scores, and greater baseline suicide item scores were significantly associated with a greater number of ECT treatments to suicidal resolution (Supplementary material Table S2). Further analysis using forward multivariate Cox regression analysis revealed that past suicide attempt and greater baseline WSAS scores were the definite predictors (Supplementary material Table S3).

Suicidality ratings significantly improved during acute treatment with ECT. About $50 \%$ of patients achieved the suicidal resolution after six ECT treatments. Patients with past suicide attempts, longer duration of the current episode, higher levels of pain, greater psychosocial functional impairment, and higher levels of suicidality were less likely to achieve suicidal resolution. Past suicide attempt may increase the likelihood of future attempts, and a suicide attempt is the strongest predictor for a completed suicide. ${ }^{5}$ Longer duration of the current episode predicted the greater number of ECT treatments to resolve suicidality. It indicates that ECT should be considered earlier in the course of treatment for depressed patients at risk of suicide. Traditionally, ECT is often recommended as a "treatment of last resort" in the real world. Therefore, more number of ECT treatments is required to resolve the suicidality if depressed patients receive ECT too late. However, more number of ECT treatments is likely to have more cognitive impairment. Baseline pain reduced the benefits of ECT to resolve suicidality. Suicide and suicidal behavior should be considered as the expression of an attempt to escape from unbearable psychological pain. Some patients may believe that it is possible to end pain by stopping consciousness. ${ }^{5}$ Psychosocial dysfunction is associated with severity of depression and suicidality. Based on the interpersonal- psychological theory of suicide, both perceived burdensomeness and the feeling of alienation from social circles are associated with suicidality. Further analysis revealed that past suicide attempt and greater psychosocial functional impairment contributed to longer time to suicidal resolution than other factors.

Patients treated with ECT were already at high risk of suicide. Fifty percent of patients achieved suicidal resolution after six ECT treatments. Combined ECT with psychotherapy could be expected to decrease the number of ECT treatments to resolve suicide risk. However, further investigation is required.

\section{Supplementary material}

To view supplementary material for this article, please visit http://dx.doi.org/ 10.1017/S1092852919000920

\section{Disclosures}

The authors declare they have nothing to disclose.

\section{REFERENCES:}

1. Fink M, Kellner $\mathrm{CH}$, McCall WV. The role of ECT in suicide prevention. J ECT. 2014; 30(1): 5-9.

2. Lin CH, Chen MC, Yang WC, et al. Early improvement predicts outcome of major depressive patients treated with electroconvulsive therapy. Eur Neuropsychopharmacol. 2016; 26(2) 225-233.

3. APA. The Practice of Electroconvulsive Therapy: Recommendations for Treatment, Training, and Privileging: A Task Force Report of the American Psychiatric Association. 2nd ed. Washington, DC: American Psychiatric Association; 2001.

4. Mundt JC, Marks IM, Shear MK, et al. The Work and Socia Adjustment Scale: a simple measure of impairment in functioning. Br J Psychiatry. 2002; 180: 461-464.

5. APA. Practice guideline for the assessment and treatment of patients with suicidal behaviors. Am J Psychiatry. 2003; 160(11 Suppl): 1-60. 